\title{
Characterising the fortified foods consumed by school-aged children $(5-12 y)$ in Ireland
}

\author{
N. Walsh ${ }^{1,2}$, J. Walton ${ }^{2}$, M. Buffini ${ }^{3}$, J.M. Kearney ${ }^{4}$, B.A. McNulty ${ }^{2}$, A. Flynn ${ }^{1}$ and L. Kehoe ${ }^{1}$ \\ ${ }^{1}$ School of Food and Nutritional Sciences, University College Cork, Cork, Ireland, \\ ${ }^{2}$ Department of Biological Sciences, Munster Technological University, Cork, Ireland, \\ ${ }^{3}$ Institute of Food and Health, University College Dublin, Belfield, Dublin, Ireland and \\ ${ }^{4}$ School of Biological and Heath Sciences, Technological University Dublin, Dublin, Ireland
}

Fortification of foods with micronutrients can represent an opportunity to increase intakes of micronutrients in population groups. Within Europe, micronutrients are permitted to be added to foods voluntarily at the discretion of the manufacturer under EU Regulation (EC) No 1925/2006 ${ }^{(1)}$. The aim of this study was to characterise the fortified foods consumed by school-aged children in Ireland including the amount and types of fortified foods and the specific nutrients added.

Analyses were based on the National Children's Food Survey II (NCFS II) (2017-18) which used a 4-day weighed food diary to collect detailed information on food and beverage consumption in a nationally representative sample of school-aged children (5-12years) in Ireland ${ }^{(2)}$. Participants were asked to provide product labels for all foods and beverages consumed during the recording period. Where product labels were unavailable, a photograph of the product label was obtained from retail outlets. Fortified foods were identified as those that had one or more micronutrients added in the ingredient list. A database of fortified foods consumed by school-aged children in Ireland was created providing detailed information on the type and brand of the food and the specific nutrients added.

A total of 278 foods were identified as fortified foods in the NCFS II food consumption database. Of these, $30 \%$ were breakfast cereals (27\% ready-to-eat breakfast cereals (RTEBC); 3\% oat cereals) and 26\% were dairy and non-dairy alternatives including milks and alternatives $(8 \%)$, fat spreads $(6 \%)$, yogurts $(9 \%)$, cheeses $(2 \%)$ and dairy desserts $(<1 \%)$. Other fortified food groups included soft drinks $(9 \%)$ cereal bars $(8 \%)$, biscuits, cakes, confectionery \& savoury snacks $(7 \%)$, breads $(5 \%)$, other foods/beverages $(6 \%)$ and components of composite dishes $(9 \%)$. The most common nutrients added to fortified foods were vitamin D $(64 \%)$, riboflavin $(53 \%)$, folic acid $(51 \%)$, vitamin B12 (44\%), niacin $(43 \%)$, vitamin B6 $(42 \%)$, thiamin $(41 \%)$, iron $(40 \%)$, calcium $(35 \%)$ biotin $(21 \%)$, vitamins E (20\%), C (14\%) and A ( $9 \%)$. Zinc, pantothenate, magnesium, iodine and vitamin K were less commonly added to foods $(\leq 4 \%)$. RTEBC were primarily fortified with vitamin D, B-vitamins (thiamin, riboflavin, niacin, B6, B12, folic acid and biotin) and iron. Milks were primarily fortified with vitamins D, E, riboflavin, folic acid and calcium and non-dairy alternative milks were fortified with vitamins D, riboflavin, B12 and calcium. Fat spreads were primarily fortified with vitamins A and D and non-dairy fat spreads were also fortified with vitamins E and B12. Yogurts were primarily fortified with vitamin D and calcium and soft drinks with vitamins $\mathrm{C}, \mathrm{B} 6, \mathrm{~B} 12$ and zinc.

This database of fortified foods shows that a wide range of foods consumed by school-aged children in Ireland are fortified with a variety of micronutrients and will be useful for policy makers and the food industry.

\section{Acknowledgements}

The National Children's Food Survey II was funded by the Irish Department of Agriculture, Food and the Marine.

\section{References}

1. European Commission (2006) The OJEU 1925/2006 ECC, L404, 26-38.

2. Irish Universities Nutrition Alliance (IUNA) (2019). The National Children's Food Survey II (NCFS II) www.iuna.net. 